\title{
ESTRATEGIAS LOCALES AL SERVICIO DE LA INNOVACIÓN: LOS CASOS DE ARANDA DE DUERO Y MEDINA DEL CAMPO (CASTILLA Y LEON) ${ }^{1}$
}

\author{
Basilio CALDERÓN CALDERÓN - José Luis GARCÍA CUESTA - Henar PASCUAL \\ RUIZ-VALDEPEÑAS - Ignacio MOLINA DE LA TORRE \\ Departamento de Geografía, Universidad de Valladolid
}

Recibido: 09/09/2009

Aceptado: 05/02/2010

RESUMEN: El propósito de este estudio es el análisis de la vertiente socio institucional de los procesos de innovación en la escala local identificando, en las ciudades de Aranda de Duero y Medina del Campo -Castilla y León-, los agentes y redes creadas ad hoc, y tratando de analizar las consecuencias que estos procesos tienen en cada lugar, no sólo en su crecimiento económico, sino también en los aspectos sociales, ambientales y territoriales.

$P A L A B R A S C L A V E$ : innovación, redes sociales e innovación, sistema regional de innovación, innovación local.

LOCAL INNOVATION STRATEGIES: THE CASE OF ARANDA DE DUERO AND MEDINA DEL CAMPO (CASTILE AND LEÓN)

ABSTRACT: The aim of this paper is to analyse the socio-institutional aspects of innovation processes on a local scale, identifying in Aranda de Duero y Medina del Campo (Castilla y León, Spain) those agents and networks created $a d h o c$, and attempting to analyse the impact of these processes in each area, not only on their economic growth, but also in social, environmental and territorial terms.

KEY WORDS: innovation, social network, territorial development, territorial innovation systems.

STRATÉGIES LOCALES AU SERVICE DE L'INNOVATION: CAS D'ARANDA DE DUERO ET MEDINA DEL CAMPO (CASTILLE-ET-LEON)

RÉSUMÉ: L'objectif de cette étude est double : il s'agit, d'une part, d'analyser l'aspect socio-institutionnel des processus d'innovation à échelle locale en examinant les agents et réseaux créés ad hoc dans les villes d'Aranda de Duero et Medina del Campo, région de Castilleet-León. Et, d'autre part, de soumettre à l'analyse les conséquences que ces processus ont eues

\footnotetext{
${ }^{1}$ El presente artículo ha sido elaborado en el marco general del proyecto coordinado: Procesos de innovación en ciudades intermedias y desarrollo territorial policéntrico, de la Dirección General de Investigación, Subdirección General de Proyectos de Investigación, Referencia: SEJ2006-14277-C04-04/GEOG.
} 
sur les différentes localités non seulement en termes de croissance économique mais aussi en termes sociaux, environnementaux et territoriaux.

MOTS-CLÉS: innovation, réseaux sociaux et innovation, système régional d'innovation, innovation locale.

ESTRATÉGIAS LOCAIS AO SERVIÇO DA INOVAÇÃO: OS CASOS DE ARANDA DE DUERO E MEDINA DEL CAMPO (CASTELA E LEÃO)

RESUMO: O propósito deste trabalho é o análise da corrente sócio institucional dos processos da inovação na escala local identificando, nas cidades de Aranda de Duero e Medina del Campo - Castela e Leão-, os agentes e redes criadas ad hoc, e procurando analisar as consequências que tem em cada lugar estes processos, não somente em seu crescimento económico, mas também nos aspectos sociais, ambientais e territoriais

PALAVRAS-CHAVES: inovação, redes sociais, e inovação, sistema regional de inovação, inovação local .

\section{INTRODUCCIÓN}

En los últimos años, prácticamente todas las regiones españolas y obviamente las mayores ciudades de sus respectivos sistemas urbanos, han desarrollado estrategias de innovación, vinculadas por lo general a la investigación y desarro1lo, apoyadas en una red de universidades, centros tecnológicos, parques científicos o parques tecnológicos; en no pocos casos, estas estrategias han olvidado o marginado el papel de los procesos innovadores de lo que podríamos denominar segundo nivel, entendiendo como tal aquellos que no son necesariamente tributarios de las estrategias de investigación amparadas por el sistema regional o nacional de ciencia y tecnología y desarrolladas por las universidades o centros de investigación públicos o privados radicados en cada territorio.

Sólo de forma excepcional es posible identificar ciudades, e incluso pequeñas ciudades como las que se analizan en este trabajo, en las que se pueden encontrar iniciativas en los dos niveles apuntados: por una parte, estrategias de innovación apoyadas en la investigación, desarrollada por grandes empresas con relativo amparo institucional -regional o provincial-, y por otra múltiples iniciativas empresariales, directamente relacionadas con la existencia de entornos locales innovadores en los que se materializa la "...cooperación entre las instituciones, asociaciones y agentes que de una u otra forma tienen presencia activa en el funcionamiento del sistema productivo" (CARAVACA, I, GONZÁLEZ, G. SILVA, R., 2003), y en los que se registran mejoras de carácter incremental o adaptativo, con las que se da cobertura a formas de innovación, ya sea en procesos o en organización, que permiten dotar de carácter competitivo a una parte de su perfil.

El objetivo de este trabajo consiste en el análisis de la vertiente socio institucional de los procesos de innovación en la escala local, identificando en dos 
ciudades medias de Castilla y León, las más pequeñas de la jerarquía urbana: Aranda de Duero y Medina del Campo, aquellos agentes y redes creadas con objeto de compensar las debilidades asociadas a su propio tamaño y singularmente a la carencia de centros de investigación básica y aplicada, tratando de valorar las consecuencias que estos procesos tienen en cada caso, no sólo en su crecimiento económico, sino también en aspectos sociales, ambientales y territoriales.

\section{UNA APROXIMACIÓN METODOLÓGICA AL ESTUDIO DE LA INNOVACIÓN EN LAS CIUDADES MEDIAS}

El modo de entender la innovación apuntado es parcialmente tributario de aquellas aportaciones teóricas, realizadas en las últimas décadas del siglo pasado, que vienen a destacar el significado de dos elementos en la génesis de actividades innovadoras: la capacidad o necesidad de las empresas para innovar, por un lado, y la influencia de las organizaciones o instituciones sobre las empresas, materializada en la creación o mejora de los denominados entornos innovadores por otro (LUNDVALL, B.A., 1992); y asumiendo al tiempo que "la innovación en organización podría ser una condición previa y necesaria para las innovaciones tecnológicas" (LAM, A., 2005). Desde esta perspectiva, en un sistema de innovación, entendido como aquel contexto en el que el conocimiento y tecnología que se genera es resultado de la interacción social con su entorno (LUNDVALL, B.A. 1992), lo verdaderamente relevante no es sólo la existencia de un conjunto de actores -empresas, instituciones de educación superior e investigación, agencias de transferencia de tecnología, cámaras de comercio, asociaciones de empresas, etc...- sino la capacidad de alcanzar un determinado tipo de relaciones asociativas, incluyendo algunas infraestructuras físicas y de relación, donde contactan los diferentes agentes: centros de trabajo, consorcios (HIRST, P. 1994, CASSON, M. 1995) y en general el territorio, que es escenario y recipiente de la innovación.

Aunque las aportaciones de la Geografía al conocimiento de los procesos de desarrollo territorial en los que están implicadas diversas formas de innovación es relativamente reciente, la calidad de las mismas viene a cubrir parcialmente la tardía incorporación a esta temática, habiéndose centrado preferentemente en el análisis de los sistemas territoriales de innovación, para destacar tanto la identificación teórica y empírica de las redes socio institucionales, como el papel de los actores locales en los nuevos espacios económicos y en general en el desarrollo territorial en España (SALOM, J. y ALBERTOS, J.M., 2009 y SALOM, J. 2003); y especialmente, las consecuencias que los procesos de innovación tienen en cada territorio, "no sólo en su crecimiento económico, sino también en 
los aspectos sociales, ambientales y territoriales" (SALOM, J. y ALBERTOS, J.M., 2009, pp. 13).

Esta perspectiva ha dado pie a nuevas aportaciones que parten de la conveniencia de abordar la innovación a otras escalas, es decir, contemplando otros agregados territoriales dotados de cierta capacidad para operar de forma cooperativa, como es el caso de las ciudades medias; en esta línea se inscriben, además de los citados, los trabajos publicados -solos o en colaboración-de R. MÉNDEZ, $(2002,2006)$ sobre estrategias y redes socio-institucionales de innovación en ciudades intermedias y sobre desarrollo territorial en ciudades medias en Castilla La Mancha, también de J. SALOM (2003 y 2009), de I. CARAVACA $(2002,2009)$ o G. GONZÁLEZ (2006) sobre los sistemas productivos locales e innovación en algunas ciudades andaluzas.

$Y$ es que, la importancia que las estrategias organizativas locales tienen en el desarrollo de diversos tipos de innovación es apreciable de forma inequívoca incluso en el borde del sistema urbano, es decir en las ciudades más pequeñas del conjunto de ciudades medias como es el caso de las ciudades objeto de estudio en este artículo: Aranda de Duero y Medina del Campo; ciudades que al no ser capitales y no formado parte de un entorno metropolitano, han consolidado un modelo de innovación alejado de la dinámica de los grandes centros urbanos y han de buscar la excepcionalidad, es decir, la explotación de su potencial endógeno, aprovechando su capacidad organizativa y las posibilidades contempladas en el sistema regional de innovación en lo que se refiere a la financiación, o la dotación de infraestructuras físicas o de gestión.

La metodología utilizada para verificar esta hipótesis ha consistido por un lado en el análisis de los instrumentos de gestión territorial disponibles en ambas ciudades, así como su grado de aplicación y operatividad deducido a través de las fuentes estadísticas regionales, así como de encuestas y entrevistas a los agentes más relevantes de cada ciudad, con los que se han construido los correspondientes sociogramas para verificar las conexiones de cada agente con los restantes del sistema local; y por otro en el análisis del conjunto de proyectos empresariales demandantes de financiación institucional en 2007 a través de ADE -Agencia de Inversiones y Servicios de Castilla y León, especialmente los proyectos de $\mathrm{I}+\mathrm{D}+\mathrm{i}$, los vinculados a la Consolidación de Estructuras de $\mathrm{I}+\mathrm{D}+\mathrm{i}$ ", los Incentivos Regionales y los incentivos a la inversión de Especial Interés; merced a todo ello se ha podido demostrar, en el caso de Medina del Campo, el carácter limitado del aprovechamiento de las ayudas e inventivos, y el mayor rendimiento obtenido por las grandes empresas radicadas en Aranda de Duero, como resultado sin duda de la mayor tradición industrial y la mayor diversificación de su tejido productivo. 


\section{DOS MODELOS DE INDUSTRIALIZACIÓN Y DESARROLLO ECONÓMICO Y TERRITORIAL: ARANDA DE DUERO Y MEDINA DEL CAMPO.}

Aranda de Duero y Medina del Campo, con 32.460 y 21.256 habitantes respectivamente, se encuentran en la base de la jerarquía urbana regional. Ambas ciudades se caracterizan por presentar una vitalidad económica relativamente importante y recientemente renovada, que ha favorecido un crecimiento de la población desde 1998, cifrado en un 6\% en el caso de Medina del Campo y un 9\% en el de Aranda de Duero y que contrasta con la acusada atonía demográfica de otros espacios de la Región. La primera, que es cabecera del área funcional de la comarca de la Ribera que supera los 50.000 habitantes, cuenta con las indudables ventajas comparativas que se derivan de la intersección en su término municipal de dos vías esenciales: la que asegura la comunicación Norte-Sur a través de la A-1 y la comunicación Este-Oeste, mediante la futura conversión de la N-122 en autovía, constituyendo por tanto un nudo estratégico en la infraestructura de comunicaciones del sur de la provincia de Burgos. Por su parte, Medina del Campo, situada al suroeste de Valladolid y próxima a la capital, es el centro de la comarca Tierra de Medina y está dotada de una alta accesibilidad y de una posición estratégica dentro de los flujos económicos del centro y suroeste de la Región a lo que hay que añadir la accesibilidad y relativa proximidad a Madrid cuya influencia es muy perceptible en la ciudad.

Figura 1: Trabajadores en el sector industrial en las ciudades de Castilla y León

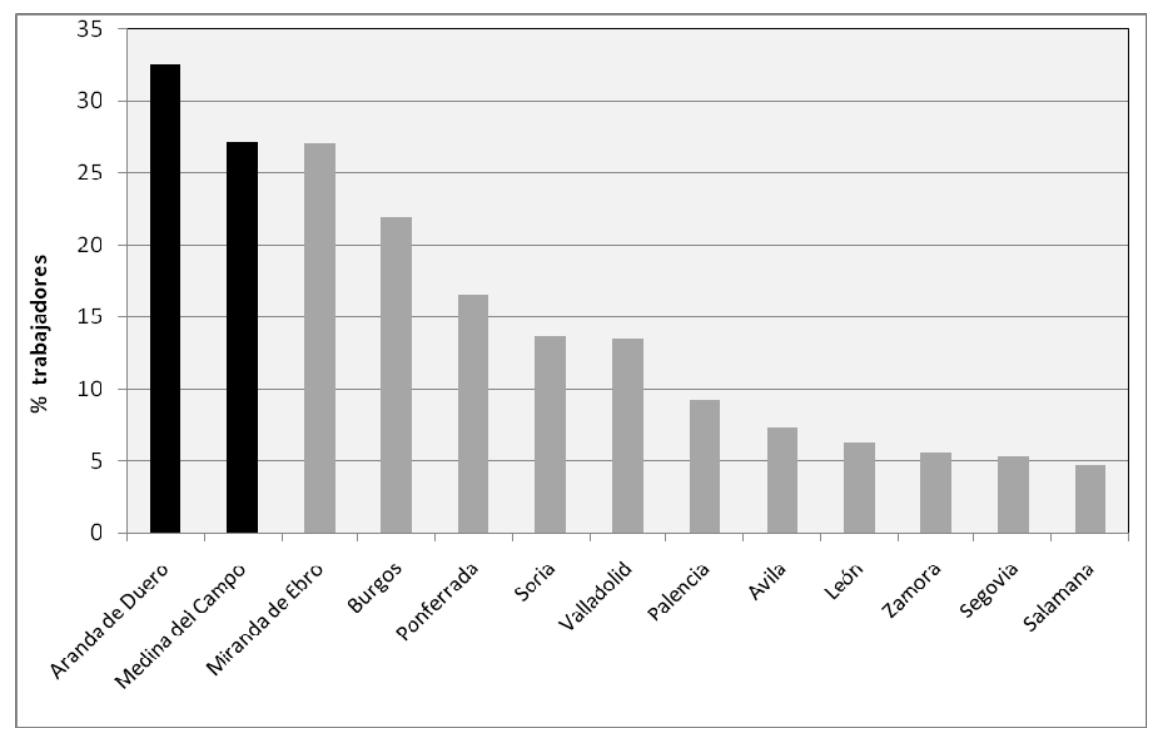

FUENTE: Tesorería General de la Seguridad Social (Diciembre de 2008) 
La estructura de la actividad de ambos núcleos urbanos muestra notables contrastes en relación con otras ciudades de la Región y, en particular, con las capitales provinciales, puesto que en Aranda de Duero y en Medina del Campo, el sector servicios registra comparativamente un menor peso específico. Así, mientras que la proporción de trabajadores en los servicios supera ampliamente el $70 \%$ en la mayoría de las ciudades castellanoleonesas, en Aranda de Duero y en Medina del Campo, el peso de los efectivos laborales en ese sector se cifra en el $63 \%$ y el $67 \%$ respectivamente.

$\mathrm{Y}$ es que, ambas ciudades se identifican como importantes centros industriales a escala regional y de hecho las actividades manufactureras constituyen uno de los principales motores de dinamización de sus economías locales. Aranda de Duero es realmente la ciudad con mayor especialización industrial de la Región, ya que un tercio de sus efectivos laborales están ocupados en establecimientos de fabricación (FIGURA 1); su tejido productivo tiene origen en la política de planificación desarrollista y más concretamente en su declaración en 1959 como Polígono de Descongestión Industrial de Madrid y en la posterior aprobación en 1965 del Plan de Industrialización de la Zona Económica de Aranda de Duero. Las repercusiones de dicho Plan fueron muy limitadas y se vieron rápidamente eclipsadas por la decisión de la multinacional francesa Michelín de instalarse en Aranda, una decisión directamente vinculada a su proximidad y accesibilidad a los principales complejos de automoción del país (Valladolid, Madrid, Barcelona) y al resto de sus factorías en España (Vitoria, Lasarte) y Francia, actuando también como factor de atracción la progresiva concentración de mano obra en el municipio procedente del entorno rural.

A la instalación de esta factoría le siguió pocos años después la implantación de una planta de la farmacéutica norteamericana Glaxo. Asimismo, y como contrapunto a estas dos filiales multinacionales, la compañía de origen local Grupo Leche Pascual se fue consolidando y proyectando en el mercado nacional e internacional a partir de pequeños negocios de ámbito comarcal en un proceso continuo de crecimiento y expansión, hasta convertirse en una de las principales compañías alimentarias de capital nacional.

Asimismo, Medina del Campo es una ciudad que registra una elevada proporción de trabajadores empleados en la industria -el 27\%-lo que la sitúa en la segunda posición en la jerarquía de especialización industrial, al mismo nivel que Miranda de Ebro (FIGURA 1). Aun siendo más antigua su vocación industrial, por estar estrechamente vinculada a la actividad ferial tradicional, no será, como en el caso de Aranda de Duero, hasta mediados del siglo XX cuando inicie su desarrollo como núcleo industrial, al instalarse alguna de las mayores empresas de la ciudad, como la compañía Ferroaleaciones; su consolidación como centro manufacturero está estrechamente vinculada a una fuerte especialización en el 
sector del mueble, a tal punto que en los años ochenta contaba ya con más de 44 fábricas y otras 18 empresas relacionadas con el sector, superando así a la actividad tradicional en la ciudad que era la fabricación de productos alimenticios. Cierto es que se trata de una industria de dimensiones muy modestas, ya que tan sólo tres empresas contaban con más de 50 trabajadores, pese a lo cual ha conformado la base productiva local y el mayor yacimiento de pequeña innovación -diseño de producto, comercialización- del municipio hasta finales del siglo XX, aprovechando la alta concentración de demanda de equipamiento del hogar que generó el crecimiento urbano regional en este periodo.

Figura 2: Tamaño medio de los establecimientos industriales

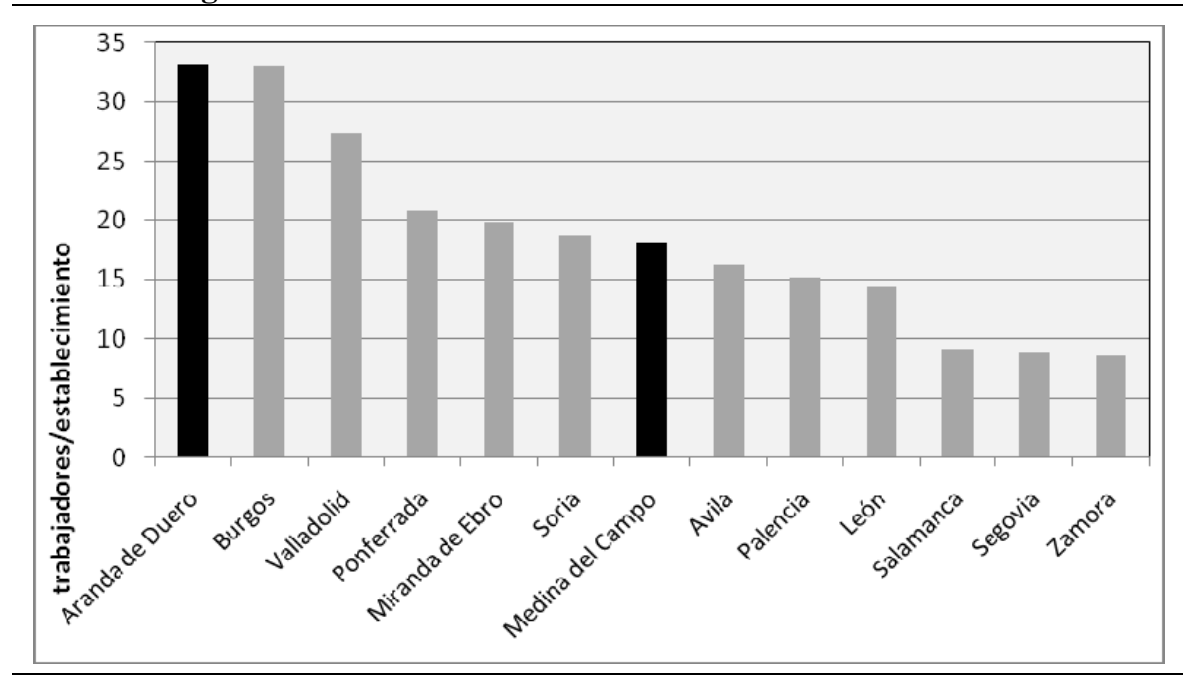

FUENTE: Tesorería General de la Seguridad Social (datos a diciembre de 2008)

El tejido productivo de ambos municipios se está densificado y especializando, contribuyendo a ello, sin duda, la renovada capacidad de movilización y atracción de iniciativas empresariales -endógenas y exógenas-y la ampliación de la superficie de suelo industrial planificado que han dado lugar a la configuración de nuevos y dinámicos espacios industriales tales como el de Prado Marina en Aranda de Duero, o los polígonos Francisco Lobato, Medina ON y Escaparate, en Medina del Campo. En estos recintos industriales están en pleno desarrollo proyectos como los de Grupo Gerardo de la Calle (materiales de construcción, edificaciones industriales, tratamiento y reciclaje de residuos de construcción), Tubos Aranda (fabricación de tubos), Dandor Sol (placas de energía solar), Todoaceros (decapado mecánico de bobinas de acero), Tecnoaranda (fabricación de torres de aerogeneradores) o Verdifresh (preparados vegetales), en Aranda de Duero; y en Medina del Campo, las nuevas instalaciones 
industriales de compañías como Gamba Natural (criadero de langostinos), Galván Sport (fabricación de instalaciones deportivas), Emina Rueda (elaboración y crianza de vinos), Calprint (impresión gráfica), Patatas Meléndez y Parmentine, (selección, envasado y transformación de patatas), IbersnacksGrefusa (producción de snacks) y ABN Pipe Systems (tuberías de polipropileno).

Respecto a sus características empresariales y sectoriales, cabe reseñar que, en Aranda de Duero, a pesar del denso tejido de pymes que conforman el sistema productivo, la presencia de grandes factorías determina que el tamaño medio de los establecimientos sea muy elevado-FIGURA 2-y que los efectivos laborales se concentren en tan sólo cinco ramas de actividad fundamentales que aglutinan el 92\% del empleo industrial -FIGURA 3-. No obstante, es en la industria química y en la fabricación de productos de caucho donde la actividad está referida casi exclusivamente a una única gran fábrica - Glaxo Smithkline y Michelín respectivamente-, en cambio, en los sectores alimentario, productos metálicos y otros productos minerales, coexisten establecimientos de gran tamaño-Grupo Leche Pascual- con centros de transformación de tamaño medio y pequeño.

Figura 3: Distribución sectorial de las empresas y el empleo industrial en Aranda de Duero

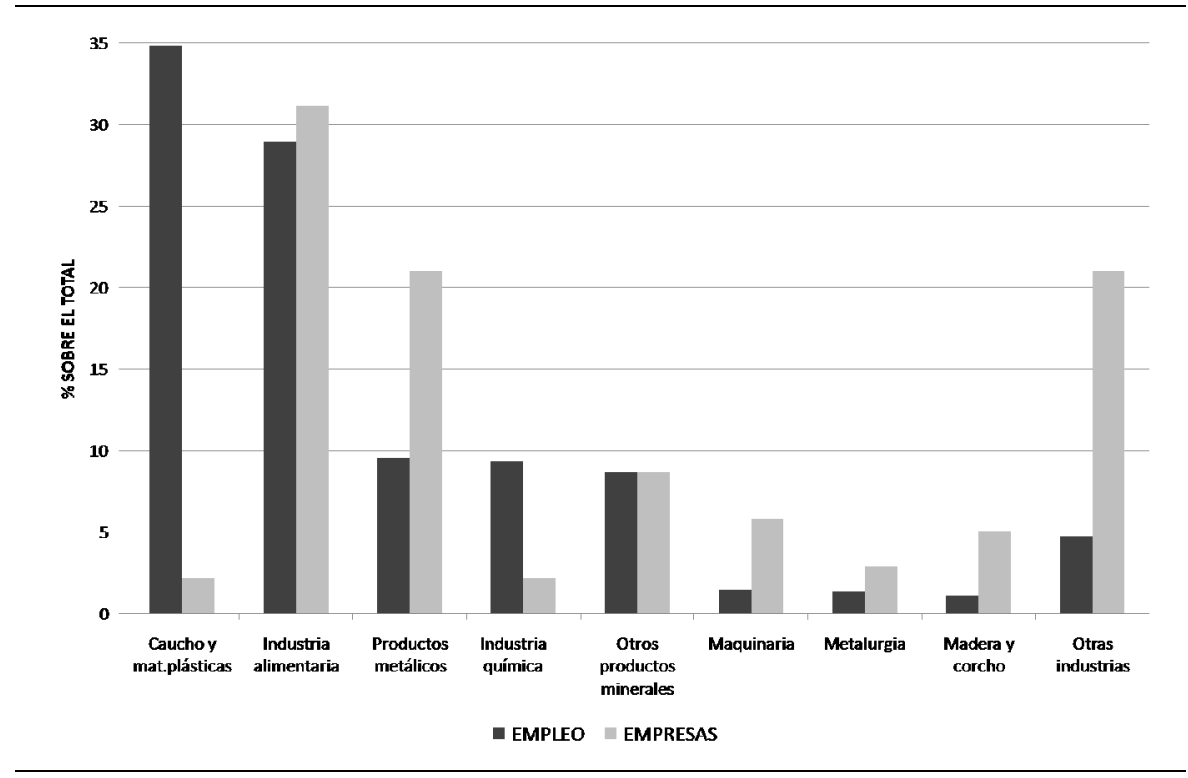

FUENTE: Tesorería General de la Seguridad Social (datos a diciembre de 2008)

También en Medina del Campo la industria alimentaria sobresale actualmente como uno de los sectores más importantes de entre los que integran su tejido 
productivo. De hecho, esta rama de fabricación, la industria del mueble y los transformados metálicos, concentran conjuntamente algo más del $70 \%$ de los efectivos laborales de la industria local-FIGURA4-. Los contrastes entre factorías de gran dimensión y pequeñas unidades de producción no son tan acusados en la industria medinense como en la arandina, pero también se observan diferencias sectoriales en el tamaño de las empresas. Así por ejemplo, si en el sector del mueble, la edición y artes gráficas o la confección, predominan los pequeños establecimientos, en la fabricación alimentaria, la metalurgia, la producción de materias plásticas o la fabricación de maquinaria y material eléctrico, el tamaño de las factorías implantadas en el municipio es bastante mayor.

Figura 4: Distribución sectorial de las empresas y el empleo industrial en Medina del Campo

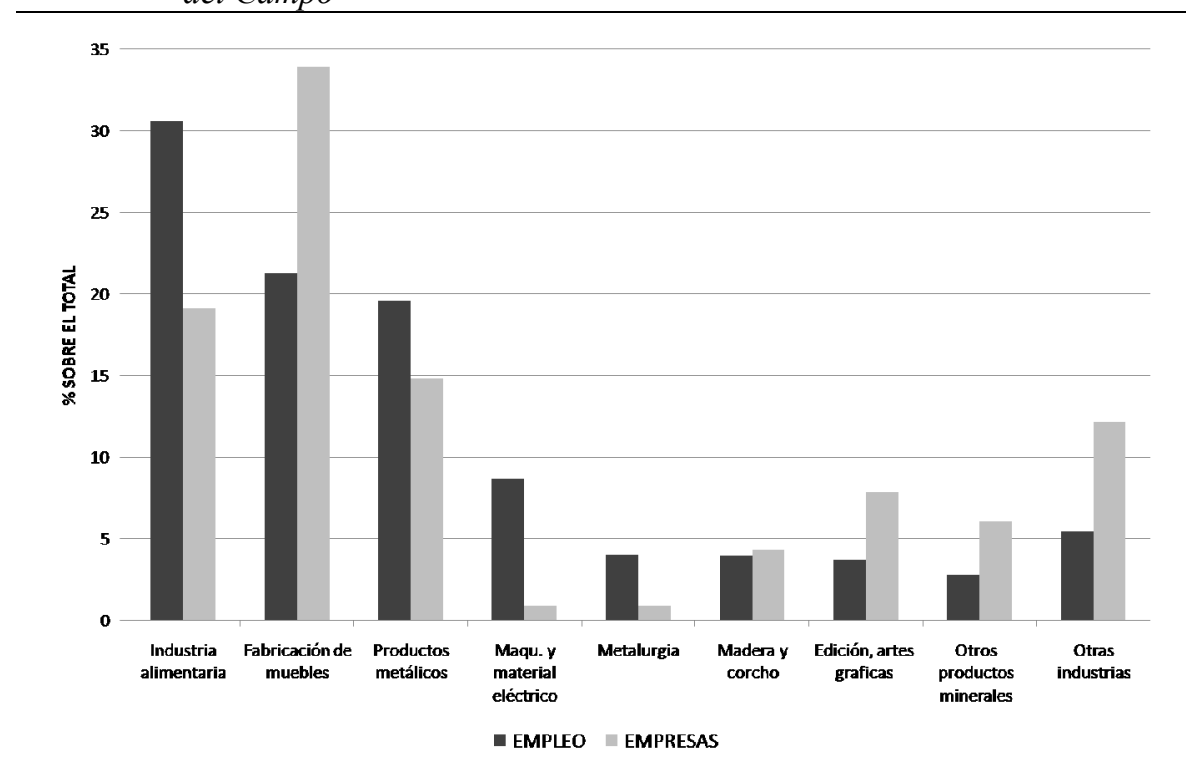

FUENTE: Tesorería General de la Seguridad Social (datos a diciembre de 2008)

Sobre esta base productiva local, conformada básicamente por sectores maduros y al margen de las grandes estrategias de $\mathrm{I}+\mathrm{D}+\mathrm{i}$ regionales, en las dos ciudades que nos ocupan se ha venido impulsando en los últimos años una suerte de cultura de innovación apoyada en iniciativas institucionales, que ha impregnado el quehacer de cuantas entidades públicas o privadas están implicadas en el desarrollo local; tanto las que se encargan de la mejora del entorno e infraestructuras físicas y sociales concebidas para tal propósito, como de aquellas esencialmente las empresas- que lo hacen efectivo merced a las mejoras introducidas en sus procesos productivos, de organización o de comercialización. 


\section{LA COEXISTENCIA DE SISTEMAS DE INNOVACIÓN EN ARANDA DE DUERO: INVESTIGACIÓN Y DESARROLLO $V S$ ASOCIACIONISMO E INNOVACIÓN.}

La dotación de infraestructuras básicas al servicio de las empresas, especialmente suelo industrial o logístico y comunicaciones e incluso la disponibilidad de múltiples ayudas institucionales no convierte per se a un entorno local en un entorno innovador ya que para alcanzar este nivel se requiere una fuete implicación y cooperación entre todos los agentes, sustentada en un "...clima de confianza mutua propiciado por sentimientos de identidad colectiva” (CARAVACA I., GONZÁlEZ G., SILVA R., 2003, pp. 108). Una identidad que, perdidas en muchos casos las referencias históricas o culturales que la nutría, debe reforzarse ahora mediante normas, instituciones, y organizaciones creadas ad hoc, relacionadas con el tejido empresarial existente y articuladas en torno a un objetivo común del que todo o una parte del grupo de una u otra forma participa, que exige de proximidad -real o virtual-y que necesita de hitos y rutinas cooperativas con las que afianzar los sentimientos de pertenencia colectivos. Ese conjunto de actores y las relaciones que entre ellos se generan, vienen a conformar el llamado capital social sin el que todo proceso innovador de base local es imposible.

\section{Generar innovación a partir de las estrategias organizativas de carácter local.}

La investigación realizada en la ciudad de Aranda de Duero ha permitido diferenciar dos grandes factores en el proceso de construcción de su entramado innovador -FIGURA 5- que podemos identificar como innovación generada e innovación captada. El primero de estos factores de innovación es el que corresponde a la innovación generada, es decir, a aquel conjunto de actividades que, en ausencia de canales de conexión directa con las grandes entidades promotoras de $\mathrm{I}+\mathrm{D}$ a escala regional -universidades, parques tecnológicos o científicos etc...-y al margen de la investigación desarrollada por las cuatro grandes empresas locales, se nutre del soporte que proporciona la creación de un entorno innovador local formado, en primer lugar, por el entramado institucional, es decir por las diversas redes socio-institucionales impulsadas por la administración local; en segundo lugar por el entramado empresarial asociativo, liderado por una asociación de asociaciones -ASEMAR-que integra a empresas funcionalmente heterogéneas; $y$ en tercer lugar por la pertenencia a un conjunto de redes temáticas de ciudades con las que se comparte perfil funcional, potencial endógeno y estrategias de desarrollo.

Por lo que respecta a lo que hemos denominado trama institucional de carácter local, según se ha comprobado en las entrevistas y encuestas semiestructura- 
das que se han realizado, el Ayuntamiento mantiene relaciones directas acreditadas mediante acuerdos, protocolos o convenios de colaboración con una extensa relación de agentes sociales, hecho que no sólo afianza las relaciones, sino que dota a las mismas de la seguridad y continuidad precisas para acometer iniciativas de las que se derivan acciones innovadoras en la propia instancia municipal, en las asociaciones y empresas que conforman el entramado organizativo y productivo local.

Figura 5: Fuentes en el proceso de construcción de una ciudad innovadora. Aranda de Duero 2009

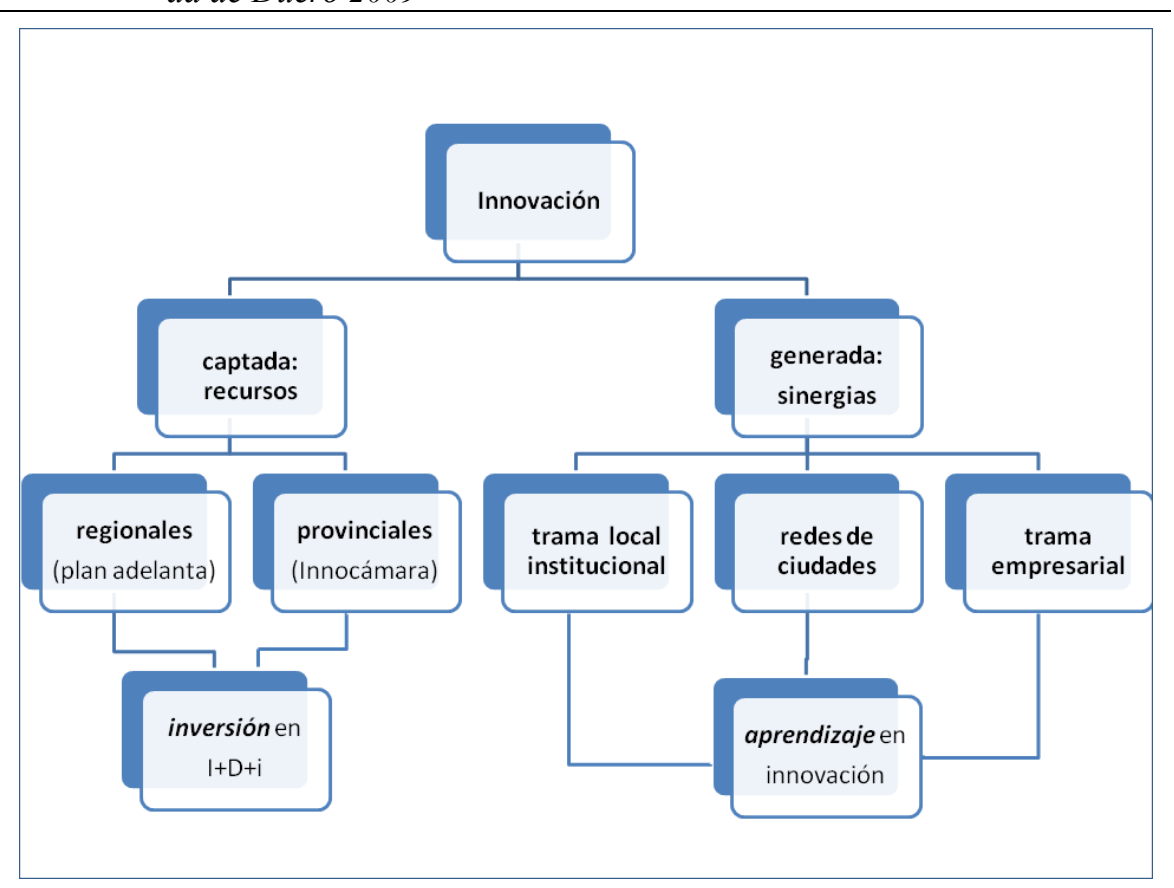

FUENTE: Elaboración propia

La gestión municipal tendente a optimizar las actividad de las empresas consolidadas y de los nuevos emprendedores con alguna capacidad de innovación ha llevado a desplegar, dentro de las inevitables limitaciones presupuestarias, un conjunto de iniciativas que podemos agrupar en dos categorías: por una parte la dotación de instrumentos -normativa-que operen a favor de la innovación y por otra la adecuación de su estructura organizativa y el impulso de las relaciones sociales en el conjunto del tejido social urbano. En el primer caso destacan las acciones relacionadas con la mejora medioambiental -Agenda Local 21con la promoción turística -Plan Director del Turismo de Aranda de Duero-y con la definición de estrategias de desarrollo a medio y largo plazo, como las 
que contiene su casi inaplicable -por ambicioso-Plan Estratégico aprobado en 2006. A ellos hay que añadir la redacción de diversos instrumentos técnicos para la gestión y promoción de Aranda de Duero como producto turístico de calidad, tales como el Plan Director de Turismo de Aranda de Duero, del año 2007, el Plan de Marketing y Actuación de la Ruta del Vino Ribera del Duero y el Plan de Dinamización del Producto Turístico "Ruta del Vino Ribera del Duero", en los que participa junto a diversos municipios de la Comarca de La Ribera.

La adecuación de la estructura organizativa municipal y el impulso de las relaciones en el conjunto del tejido social urbano constituye, como hemos indicado, el primer pilar institucional en la creación de un entorno innovador local; en esa estrategia lo realmente determinante no es tanto el organigrama municipal que atiende las necesidades de gobierno básicas o esenciales, sino aquellas secciones que tiene un carácter creativo, contemplado éste tanto desde su propia estructura organizativa, como desde el punto de vista de las acciones que desarrolla.

Para hacer frente a los nuevos requerimientos urbanos, el municipio se ha dotado, entre otras, de una concejalía de Medio Ambiente, Parques y Jardines y una oficina comarcal de Medio Ambiente, una concejalía de Desarrollo Económico y Ferias, a la que está asociada una oficina de Promoción y Desarrollo, una concejalía de Turismo, de la que depende la promoción de uno de los mayores potenciales endógenos: el vino, promocionado tanto como recurso económico o como recurso patrimonial, recuperando y habilitando para su visita turística, una parte de las más de 120 bodegas censadas en el municipio. Y, finalmente, una singular concejalía de Hermanamientos que gestiona los contactos con las 5 ciudades hermanadas: Miranda do Douro, Langen, Roseburg, Salon de Provence, y Santa Cruz de Tenerife, si bien estos no pasan de ser hasta el momento contactos protocolarios e intercambios programados de grupos de escolares o delegaciones oficiales.

La actividad que esta estructura municipal permite desarrollar está en la base del impulso creativo que dota de personalidad y capacidad para la innovación a la ciudad de Aranda de Duero, ya que además de gestionar parte de las acciones con mayor proyección exterior, asegura su presencia y participación en las redes de ciudades de las que deriva una parte su capacidad de aprendizaje e impulso innovador; tal es el caso de Acevin,-Asociación Española de Ciudades del Vino, creada en 1994 e integrada por 50 Ayuntamientos, 16 Comarcas y 3 Diputaciones, que forman parte de 27 provincias, 14 CC.AA. y 25 Denominaciones de Origen Vinícolas y una identificación geográfica protegida-. Asimismo, Aranda de Duero participa en la Red Europea de Ciudades del Vino Recevin-, que fue creada en 1995 con la intención de ser el interlocutor de las 
asociaciones de ciudades del vino de Europa con los organismos de decisión europeos, para la realización de proyectos que se basen en el desarrollo local: cualificación y formación profesional, el apoyo a la creación de empresas de carácter innovador, con componente tecnológico o para personas y colectivos de difícil inserción laboral.

Figura 6: Entramado asociativo empresarial integrante de la Asociación de empresarios de Aranda y la Ribera ASEMAR

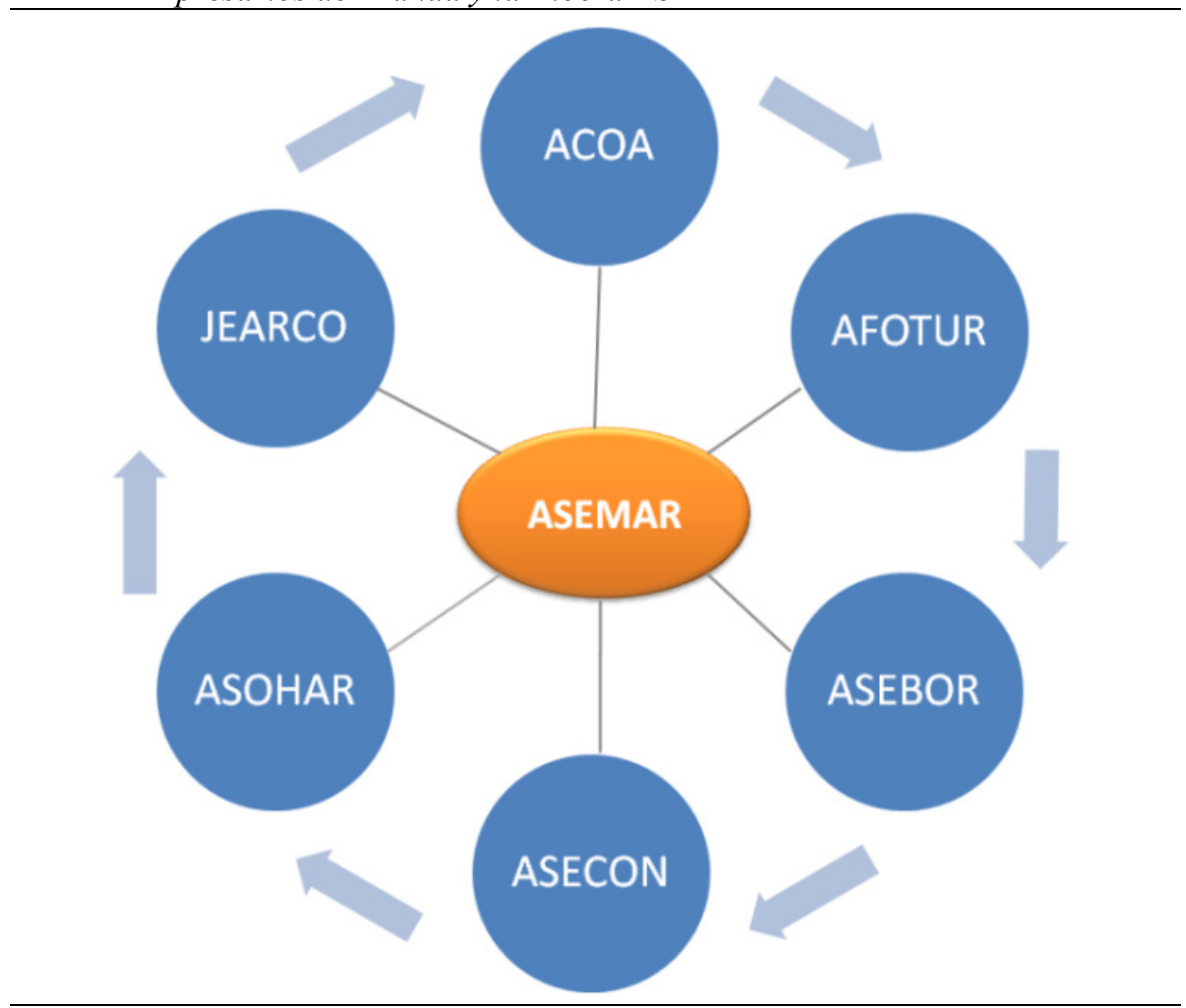

FUENTE: Elaboración propia

El tercer pilar en la estrategia orientada a generar innovación está representado por el entramado asociativo empresarial en Aranda de Duero -ver FIGURA 6-; se trata de una estructura que tiene como núcleo la asociación de empresarios de Aranda y la Ribera, creada en 1981, inicialmente como una delegación de la Confederación de Asociaciones Empresariales de Burgos -FAE-, adhiriéndose a ella, con el tiempo hasta alcanzar un total superior a los 400 asociados, empresas pertenecientes a su vez a diversas asociaciones sectoriales como ACOA-CCA, Asociación de Comerciantes de Aranda y Centro Comercial 
Abierto; ASOHAR, Asociación de Hostelería de Aranda y la Ribera; ASEBOR, Asociación de Bodegueros de la Denominación de Origen Ribera del Duero; ASECON, Asociación de Empresarios de la Construcción; AFOTUR, Asociación para el Fomento del Turismo Ribereño; y en los últimos meses de 2008, JEARCO, Asociación de Jóvenes Emprendedores, de carácter transversal, ya que el elemento aglutinador no es la actividad, como en el resto de asociaciones, sino la condición de joven empresario.

Los objetivos de esta estructura asociativa abierta están directamente relacionados con la promoción del desarrollo industrial y comercial, obviamente, pero también con el fomento de contactos y colaboraciones con las entidades territoriales, provinciales, nacionales y extranjeras de análoga naturaleza y finalidad; dicho de otra manera, esta asociación de asociaciones, de carácter marcadamente horizontal, se postula para desempeñar un papel complementario o en su caso subsidiario del que teóricamente corresponde a las administraciones en sus diversos niveles, por lo que se ha convertido en una pieza esencial del entramado básico en las estrategias de aprendizaje e innovación que se registran en la ciudad de Aranda de Duero.

Todas las asociaciones mantienen contactos entre si a través de Asomar, siendo muy variado el resultado de esta colaboración interna; en términos generales el objetivo de esta asociación no es otro que aglutinar todas las iniciativas empresariales relacionadas con la actividad económica de la ciudad de Aranda de Duero. Para cumplir con esta función pone en contacto a todos los asociados entre si ya sea mediando coyunturas como la celebración de eventos, o ya sea de forma regular a través de la mejora de los canales de información, la coordinación de cursos o jornadas formativas, la gestión de subvenciones, la planificación, comunicación y relaciones públicas en general; y para cumplir con estos objetivos dispone de sede propia, personal especializado, edita una revista de periodicidad bimensual y dispone de página web propia.

El segundo de los factores señalados - ver FIGURA 5-, es la innovación captada, una actividad que se nutre de los recursos que cada ciudad puede obtener del exterior, y a la que se llega fundamentalmente por dos vías: en primer lugar mediante la participación en proyectos de apoyo a iniciativas innovadorasemprendedoras promovidas por la administración regional o provincial, como es el programa Adelanta que coordina la Agencia de Inversiones y Servicios de Castilla y león -ADE- o bien, en segundo lugar, mediante los programas que lideran las Cámaras de Comercio, orientados a la promoción empresarial y al estímulo de la innovación en el tejido de las pequeñas y medianas empresas locales. 


\section{La captación de recursos para innovar: una estrategia al alzance la las grandes empresas locales.}

En paralelo a la actividad asociativa privada descrita, de la que se derivan notables ganancias en aprendizaje, la ciudad de Aranda participa de una gran parte de las iniciativas que permiten obtener recursos con los que financiar las estrategias empresariales de $\mathrm{I}+\mathrm{D}$; de este factor son en gran parte responsable aquellas grandes empresas que operan de forma relativamente independiente, en un contexto dominado por la pequeña y mediana empresa ya que el $81 \%$ de las que están implantadas en la ciudad no alcanzan siquiera la decena de trabajadores.

Sólo cuatro empresas, que corresponden al umbral de mayor dimensión, son responsables de dos tercios del empleo ocupado en la industria de Aranda, y aunque tres de de ellas Michelín, GlaxoSmithKline, Grupo Leche Pascual y el Grupo Gerardo de la Calle podrían tener una entidad más o menos comparable, hay un rasgo que introduce una evidente diferenciación como es el hecho de que las dos primeras son factorías de empresas multinacionales, es decir, establecimientos con funciones de fabricación que dependen de decisiones externas y en las que el núcleo fundamental de las actividades de I+D está localizado en la sede central de la empresa matriz, mientras que las dos restantes, de origen local, tienen concentradas en la ciudad de Aranda de Duero las principales funciones de dirección, gestión, administración, comercialización e I+D, pese a lo cual es esta una actividad que representa un porcentaje muy pequeño $-9,7 \%$ en el conjunto de las subvenciones concedidas por la Agencia de Desarrollo Ade- dentro del Plan Adelanta 2007 para el conjunto de la ciudad de Aranda -CUADRO 1-.

El grueso de las ayudas que proporciona la Junta de Castilla y León a través del citado organismo se concentra en los Incentivos Regionales que suman el $66,7 \%$ de las solicitudes de ayudas resueltas en 2008, y en los proyectos de Especial Interés -el 22\% - directamente relacionados con la creación en el polígono industrial Prado Marina de un pequeño cluster relacionado con la fabricación de bienes de equipo destinados a la producción de energía eléctrica eólica y solar, integrado por nuevas empresas como Dandor Sol S.L. que será primera factoría española de fabricación de placas de energía solar, Tecnoaranda, que pondrá en marcha un innovador proceso de fabricación de bobinas de chapa destinadas al desarrollo integral de torres eólicas, o las empresas Todoaceros y Aceros de China S.L-Chinaceros-.

Es cierto no obstante que, en el momento presente, nos encontramos sólo ante una simple "concentración geográfica de empresas e instituciones interconectadas en una esfera de actividad específica" (PORTER, M. 1998, p.78) y, aunque 
que esta concentración puede nutrirse de la actividad desarrollada por empresas parcialmente complementarias ya existentes en Aranda -especialmente las metalúrgicas-, ubicadas en el polígono vecino de Allendeduero, puede tener algunas limitaciones para su configuración como un cluster propiamente dicho al carecer de relaciones cercanas con instituciones gubernamentales, universidades, centros de investigación etc..., siendo éstas un elemento inherente al mismo según la conceptualización a uso de esta singular concentración empresarial y especialización territorial (PORTER, M., 1998).

Como es lógico suponer, las actividades de I+D no son habituales entre las pequeñas firmas manufactureras que integran la mayor parte del tejido fabril de la ciudad, con la excepción de dos empresas, que ejemplifican en sus respectivos sectores, el esfuerzo innovador de las pymes en Aranda de Duero: el Grupo Gerardo de la Calle, comprometido con la investigación y desarrollo pues no en vano es receptor de una cuarta parte de las subvenciones que se conceden para desarrollar proyectos de I+D -CUADRO 1-; pertenece al sector de materiales y servicios a la construcción y cuenta con una plantilla de más de 200 trabajadores distribuidos entre las empresas Hormigones y Excavaciones Gerardo de la Calle, Artepref, Calferman, y Desarrollos Industriales Prado Marina, que está encargada de la gestión del polígono del mismo nombre. Y en el sector metalúrgico, sobresale la compañía Industrias Metalúrgicas Esgueva-IMESA-, que cuenta con 80 empleados y constituye un centro de producción innovador ya que dispone de un equipo de investigación y desarrollo y una oficina técnica para el diseño de proyectos industriales.

La infraestructura local al servicio de la innovación presenta, no obstante, una importante limitación como es su desvinculación con la actividad investigadora básica, salvo la realizada por las grandes empresas, que atienda, en colaboración con la Universidad y otros centros de investigación, las necesidades en materia de innovación en el sector económico clave en la ciudad y comarca como es el sector agroalimentario; por fortuna esta carencia está parcialmente resuelta gracias a la actividad desplegada por tres entidades que constituyen el nexo entre el tejido empresarial innovador y los organismos que la promueven y financian: en primer lugar la Fundación Michelin Desarrollo, responsable de la implantación de cinco empresas: Verdifresh, Consonni, Carrozados Industriales Rojo, Chinchurreta, y el call center Digitex, con las que se han generado, en una primera fase, 440 empleos y sus proyectos de expansión prevén superar los 600 . En segundo lugar desempeña una función relevante el Instituto Tomás Pascual Sanz para la Nutrición y la Salud, que ha suscrito acuerdos de colaboración con diversas entidades -Universidades, centros de investigación etc.-para la cooperación en proyectos de investigación y de formación en el campo de la dietética, la alimentación, la seguridad alimentaria y la salud pública; y finalmente el Centro Tecnológico Alimentario -CTACYL- impulsado por 
ASEMAR, que se encontraba aún, en 2009, en fase de constitución, y que está llamado a constituirse en el nexo de unión entre la investigación y la innovación en una ciudad que, al igual que sucede en Medina del Campo ha vivido relativamente desconectada de tales estrategias hasta principios del siglo XXI.

Cuadro 1: Plan Adelanta. Proyectos de especial interés, incentivos a la inversión, incentivos regionales e $I+D$ resueltos en 2008 . Aranda de Duero

\begin{tabular}{lrrr}
\hline \multicolumn{1}{c}{ Actividad } & $(1)^{*}$ & $(2)$ & $(3)$ \\
\hline Otros procesos de la transformación del hierro y del acero & EI & 10,024 & 2,105 \\
Fabricación de maquinas-herramienta & II & 0,425 & 0,043 \\
Industria de la construcción de maquinaria y equipo mecánico & II & 0,474 & 0,038 \\
Industria de la construcción de maquinaria y equipo mecánico & II & 0,502 & 0,075 \\
Fabricación de especialidades farmacéuticas & IR & 30,974 & 3,097 \\
Industrias lácteas & IR & 82,545 & 3,302 \\
Fabricación de elementos de hormigón para la construcción & I+D & 0,309 & 0,066 \\
Fabricación de elementos de hormigón para la construcción & I+D & 0,633 & 0,142 \\
Fabricación de especialidades farmacéuticas & I+D & 1,703 & 0,426 \\
Fabricación de estructuras metálicas y sus partes & I+D & 0,595 & 0,158 \\
Instalaciones eléctricas & I+D & 0,104 & 0,038 \\
Otras actividades empresariales & I+D & 0,064 & 0,016 \\
Servicios técnicos de ingeniería & I+D & 0,104 & 0,037 \\
Transporte de mercancías por carretera & I+D & 0,228 & 0,050 \\
\hline Total & & 128,681 & 9,593 \\
\hline
\end{tabular}

(1), Línea de ayuda; (2), Inversión aceptada, millones de €; (3), Subvención concedida, millones de $€$.

*EI, Especial Interés; II, Incentivos Inversión; IR, Incentivos Regionales; I+D, Proyectos de I+D

FUENTE: ADE Castilla y León. 2009

\section{EL PAPEL DE LA ADMINISTRACIÓN LOCAL EN LA ESTRA- TEGIA DE DINAMIZACIÓN DE MEDINA DEL CAMPO: UNA VISIÓN INTEGRAL E INNOVADORA}

El carácter territorialmente selectivo de los procesos de innovación hace que las áreas rurales, con su dinámica económica y demográfica general, queden generalmente marginadas con la excepción de algunos núcleos urbanos pequeños y medios que concentran gran parte de las actividades y la población. Como indica la Estrategia Territorial Europea (1999), "en las regiones rurales con dificultades, sólo estas ciudades están en condiciones de proporcionar infraestructuras y servicios para las actividades económicas de la región y de facilitar el acceso a mercados de trabajo más importantes", amparándose, en ocasiones, en la existencia de procesos de innovación que han posibilitado la competitividad de su entorno socio-productivo.

Los diferentes estudios de caso, como el que nos ocupa, han demostrado que estos procesos de innovación no siempre tienen una relación estrecha con el sistema de investigación y desarrollo de su espacio regional de referencia, de tal 
forma que no es la incorporación de tecnología la variable que define mejor el modelo de innovación; a mayor abundamiento, las ramas sobre las que se ha asentado el crecimiento económico desarrollan productos con una tecnología ya madura, y en las que apenas se producen innovaciones tecnológicas radicales. En una de las áreas estudiadas, como es el de los centros urbanos del triángulo Medina del Campo-Cuéllar-Arévalo, su potencial económico se ha centrado en ramas industriales como la madera, los muebles, el textil o la alimentación, y su entramado empresarial, dominado fuertemente por la pequeña empresa, ha tenido generalmente dificultades financieras a la hora de realizar importantes inversiones en tecnología debido, además a que ha existido un desconocimiento sistemático de las posibilidades ofrecidas desde el sistema regional de innovación, que alejan a estas ciudades de la I+D.

La limitada cuantía de las subvenciones otorgadas por la Ade para el desarrollo de actividades de $\mathrm{I}+\mathrm{D}+\mathrm{i}$, recogidas en el CUADRO 2, es el reflejo de la escasez de procesos de innovación llevados a cabo en el municipio medinense. De hecho, la única empresa con un volumen de inversión y de subvenciones concedidas en 2008 es la bodega EMINA, situada en el polígono Escaparate, que concentra el $92 \%$ de la inversión aceptada, y el $83 \%$ de las subvenciones concedidas. Al margen de esta empresa, el resto de actividades subvencionadas se caracterizan por su reducida cuantía y su limitado valor como estrategias de innovación, sobre todo en algunas ramas tan importantes en el municipio como la fabricación de muebles o la transformación de productos agroalimentarios.

El reducido tamaño de las empresas del municipio dificulta la puesta en marcha de sistemas propios de innovación, y su estructura empresarial carece de masa crítica para el desarrollo de actividades de investigación y desarrollo de base local. De ahí que el papel de la administración local en Medina del Campo sea esencial a la hora de analizar la puesta en marcha de estrategias de desarrollo territorial, sobre las que se vertebran las iniciativas locales más significativas. Frente al mayor peso de las empresas o las asociaciones empresariales del caso de Aranda de Duero, en Medina del Campo el liderazgo de los procesos de desarrollo se sitúa sobre el gobierno local.

En este sentido, el ayuntamiento medinense ha optado por una visión integral de los procesos socioeconómicos del municipio, a partir de la definición de un conjunto amplio de planes de desarrollo que los vertebran, y, de forma paralela, ha desarrollado modelos de gobernanza participativa para su diseño, ejecución y control, lo que lo sitúa como una iniciativa innovadora a escala regional y nacional. Este proceso se ha venido desarrollando a partir del año 2000, tras la puesta en marcha del Servicio de Desarrollo Local del ayuntamiento, ya que han sido los encargados, por un lado, de ordenar los procesos participativos, y, 
por otro, de definir las líneas básicas de los planes de desarrollo que luego son aprobados.

Cuadro 2: Plan Adelanta. Proyectos de especial interés, innoempresa, incentivos regionales e I+D resueltos en 2008. Medina del Campo

\begin{tabular}{lcrr}
\hline \multicolumn{1}{c}{ Actividad } & $(1)^{*}$ & $(2)$ & $(3)$ \\
\hline Comercio al por menor de calzado y artículos de cuero & AI & 7,800 & 1,950 \\
Fabricación de artículos cerámicos de uso domestico y orna- & & & \\
mental & AP & 4,118 & 2,471 \\
Comercio al por menor & E & 25,650 & 11,286 \\
Revestimiento de suelos y paredes & E & 13,936 & 6,271 \\
Elaboración y crianza de vinos & EI & $5.344,260$ & 427,541 \\
Elaboración y crianza de vinos & IR & $9.275,466$ & 742,037 \\
Fabricación de otros muebles & INN & 41,296 & 20,648 \\
Carpintería metálica cerrajería & INT & 3,763 & 1,430 \\
Comercio por menor muebles; aparatos iluminación y otros artí- & & & \\
culos para el hogar & INT & 1,275 & 0,485 \\
Fabricación de calzado & INT & 25,903 & 10,361 \\
Fabricación de muebles & INT & 6,633 & 2,521 \\
Fabricación de muebles & INT & 58,534 & 23,413 \\
Comercio al por mayor de frutas, patatas y verduras & I+D & 652,590 & 143,570 \\
Transporte de otras mercancías por carretera & SI & 33,820 & 10,146 \\
\hline Total & & $15.495,042$ & $1.404,129$ \\
\hline
\end{tabular}

(1), Línea de ayuda; (2), Inversión aceptada, miles de €; (3), Subvención concedida, miles de $€$.

*AI, Artesanos Inversión; AP, Artesanos Perfeccionamiento; E, Emprendiendo; EI, Esoecial Interés; IR, Incentivos Regionales; INN, Innoempresa: Planes Estratégicos; INT, Internacionalización; I+D, Proyectos de I+D; SI, Sociedad de la Información FUENTE: Ade Castilla y León. 2009

En cuanto a la apuesta por la gobernanza participativa, el caso de Medina se caracteriza por la definición de diferentes modelos de participación, en función de los objetivos pretendidos. Pueden distinguirse dos tipologías básicas, según el tipo de miembros que participan: los modelos institucionalizados, en los que los integrantes forman parte de colectivos significativos; $y$ los modelos universales, en los que cualquier ciudadano puede participar en el proceso. Dentro del primer caso, desde 2002 se han puesto en marcha foros de participación para la aprobación del Pacto Local por el Empleo Sostenible, el Plan Estratégico de Desarrollo Medina 21, el Plan de Dinamización Turística, el Centro Comercial Abierto, el Centro de Iniciativas Turísticas, etc., de los que son partícipes de manera sistemática el Ayuntamiento y los grupos políticos municipales, los sindicatos, las asociaciones empresariales, las instituciones supramunicipales con sede en Medina, asociaciones culturales y sociales, etc. En unos casos, como el Pacto Local por el Empleo Sostenible, firmado en 2005, la participación supone un compromiso político claro, y forman parte de él los agentes sociales más significativos del municipio, como se aprecia en la FIGURA 7. En otros, como el 
Centro Comercial Abierto o el Plan de Dinamización Turística, los colectivos participantes tienen un sesgo claro, en función del objetivo pretendido, y su papel se sitúa más en la fase de diseño que en el de ejecución.

Figura 7: Firmantes del Pacto Local por el Empleo Sostenible (2005) por tipología de agentes. Ayuntamiento de Medina del Campo, Plan de Acción Local por el Empleo (PALE)

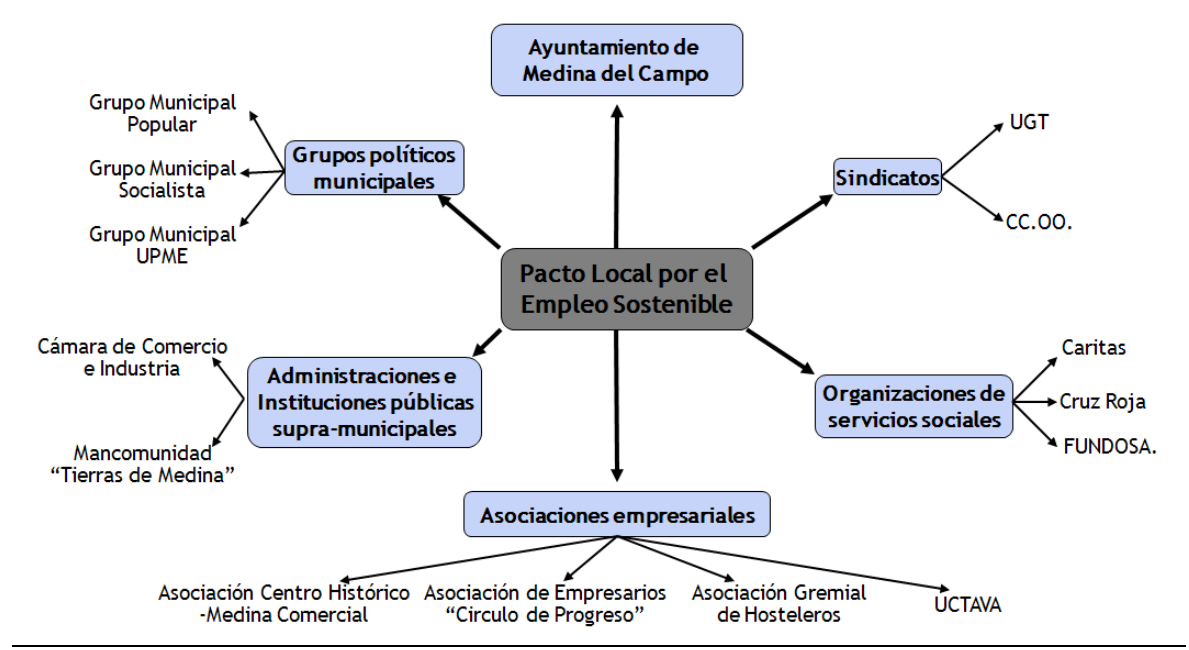

FUENTE: Elaboración propia

En cualquier caso, no se pueden separar los procesos de gobernanza participativa de la apuesta del ayuntamiento medinense por los planes de desarrollo, como modelo sobre el que definir las estrategias territoriales. Así, aspectos fundamentales en la mejora de las condiciones competitivas de Medina del Campo en su contexto territorial, como los nuevos desarrollos industriales antes señalados (Francisco Lobato, Escaparate, Medina ON), la creación de una oferta turística más amplia e integrada, la puesta en marcha del primer centro comercial abierto de Castilla y León, o la creación de un vivero de empresas forman parte de los diferentes planes de desarrollo de Medina del Campo.

De hecho, el análisis de la planificación llevada a cabo en esta ciudad demuestra, en primer lugar, la existencia de un muy elevado número de instrumentos de desarrollo, elaborados en su mayor parte en el periodo 2002-2007, y que han quedado reflejados en la FIGURA 8. El papel central de la planificación lo ostenta la Agenda 21 Local, aprobada en 2002, con tres líneas básicas de acción: social, ambiental y económica.

Por otro lado, y de forma paralela, también se ponen en marcha sistemas de participación ciudadana directa, como sucede en el diseño de la Agenda 21 Lo- 
cal, en la definición de los objetivos del Plan Medina 21, o en la Comisión Ciudadana de Participación y Seguimiento de este último plan. La importancia de estos modelos participativos estriba, por un lado, en la apuesta del gobierno local por la apertura de los planes de desarrollo a toda la población, intentando recabar el mayor apoyo popular posible antes de su aprobación; y, por otro, en la elección concreta de líneas de actuación por parte de los participantes, lo que ha dotado a estos sistemas de un valor real para la puesta en marcha de los planes de desarrollo y ha provocado una asistencia media relativamente elevada. Así, las tres mesas de desarrollo para la elaboración del Plan Medina 21, dedicadas a empleo e industria, comercio y turismo, y habitabilidad y participación ciudadana, han contado con una participación próxima a las 30 personas por sesión, y de sus deliberaciones ha salido la definición de los 10 proyectos fundamentales del Plan Estratégico, recogidos en el CUADRO 3. El análisis de esta lista demuestra la diversidad de proyectos básicos por los que han apostado los participantes, así como el carácter integral del proceso de desarrollo territorial, apoyando tanto la puesta en marcha de un Área de Rehabilitación Integrada, como la mejora de la calidad ambiental, la dinamización económica o la mejora de la gobernanza.

Cuadro 3: Plan Estratégico Medina 21. Proyectos estratégicos definidos por las comisiones ciudadanas

\begin{tabular}{|c|c|c|}
\hline$N^{o}$ & Proyecto & Mesa \\
\hline 7 & Área de Rehabilitación Integrada & Empleo e Industria \\
\hline 31 & Centro de Iniciativas Turísticas & Comercio y Turismo \\
\hline 46 & Plan de Movilidad & $\begin{array}{l}\text { Habitabilidad y Participación ciuda- } \\
\text { dana }\end{array}$ \\
\hline 39 & Creación de un Recinto Ferial & Comercio y Turismo \\
\hline 43 & $\begin{array}{l}\text { Campaî } \\
\text { ción sob }\end{array}$ & $\begin{array}{l}\text {-Habitabilidad y Participación } \\
\text { sdana }\end{array}$ \\
\hline & Recuperación de cañadas & Comercio y Turismo \\
\hline 9 & Puntos Limpios en Áreas Industria & dustria \\
\hline 57 & & $\begin{array}{l}\text { Habitabilidad y Participación ciuda- } \\
\text { dana }\end{array}$ \\
\hline 13 & Centro de Dinamización del Mueble & Empleo e I \\
\hline 69 & Reforma de la Plaza Mayor & $\begin{array}{l}\text { Habitabilidad y Participación ciuda- } \\
\text { dana }\end{array}$ \\
\hline
\end{tabular}

FUENTE: Elaboración propia a partir de Ayuntamiento de Medina del Campo. 2008

De este documento marco, y en coordinación con él, el Ayuntamiento de Medina ha ido definiendo diferentes planes de desarrollo de carácter sectorial, algunos en el seno de la propia A21L, como el Plan de Acción Local por el Empleo, el Plan de Juventud o el Plan de Igualdad de Oportunidades, y otras de forma externa pero interrelacionada (Plan de Dinamización Turística, Plan Es- 
tratégico de Servicios Sociales, etc.). Incluso el Plan General de Ordenación Urbana, aprobado definitivamente en 2008, se plantea como un instrumento esencial para la consecución de los objetivos de la A21L, en lo referente a creación de suelo industrial, movilidad urbana, calidad urbanística o renovación del centro histórico.

Ahora bien, el análisis de una estructura de planes de desarrollo tan compleja ha revelado la existencia de duplicidades en algunas medidas, y de excesiva sectorialización de las medidas en otros casos. De ahí que, a partir de 2007 y tras la evaluación de los resultados de la etapa anterior, la apuesta del gobierno local ha sido la creación de un único plan estratégico de desarrollo, basado en la metodología de trabajo de la Agenda 21 Local, y que cuente con una visión mucho más operativa. La aprobación del ya mencionado Plan Estratégico de Desarrollo "Medina 21", a partir de las indicaciones realizadas en sus mesas de preparación, es el resultado final desde el punto de vista de la planificación, si bien aún están comenzando a promoverse sus iniciativas.

Figura 7: Estructura de los planes de desarrollo en torno a la Agenda 21 Local. Ayuntamiento de Medina del Campo

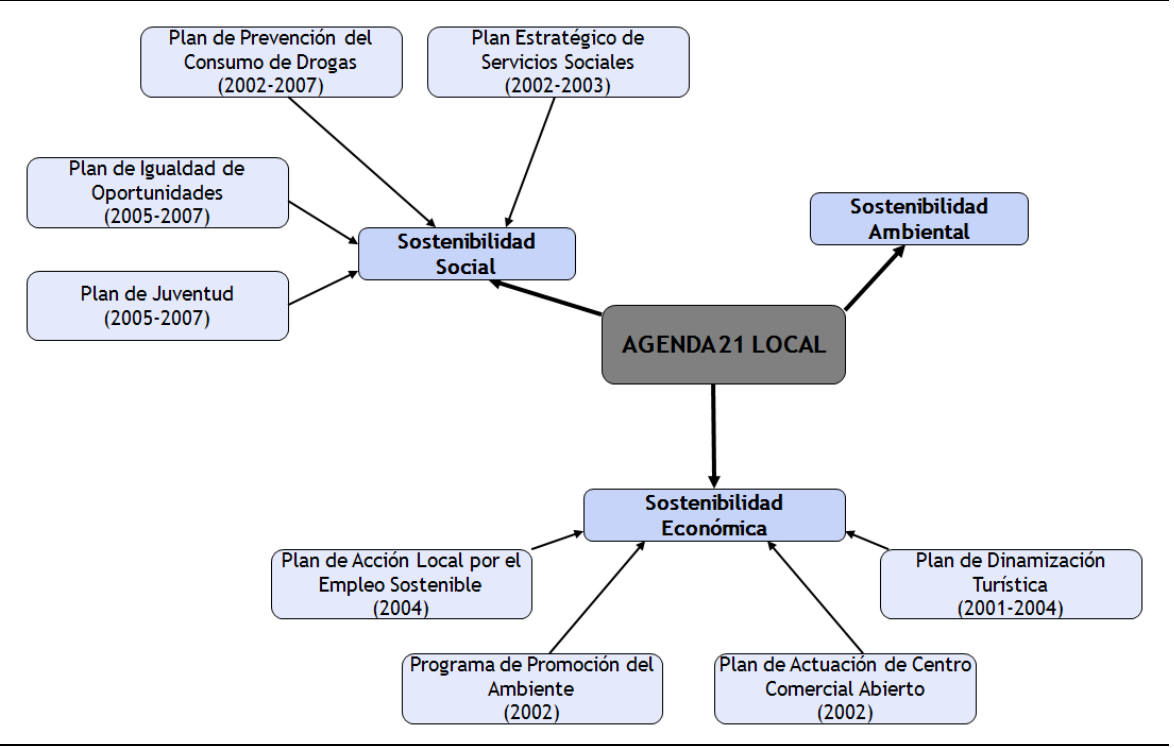

FUENTE: Elaboración propia

El resultado del liderazgo del gobierno local en los procesos de desarrollo de Medina del Campo no puede medirse únicamente en términos de mejora de los sistemas de innovación local, en la medida en que los caracteres socioeconómicos del núcleo, tanto en lo referente al sistema productivo como a las caracterís- 
ticas de los recursos humanos, son escasamente competitivos en su contexto territorial, dada su proximidad relativa a grandes áreas urbanas nacionales o regionales. Pero, en conjunto, los rendimientos obtenidos en los diferentes planes de desarrollo pueden valorarse de forma positiva porque se ha ido definiendo un proyecto de ciudad en Medina del Campo. Como resultado de las estrategias de desarrollo puede señalarse la mejora de cinco dimensiones básicas del núcleo de Medina, como son el comercio (del que el Centro Comercial Abierto y la puesta en marcha de la Asociación de Comerciantes "Centro Histórico. Medina Comercial" son los hitos básicos), el turismo - que, como en el caso anterior, tiene como pilares el Plan de Dinamización Turística y las iniciativas para poner en marcha el centro de iniciativas turísticas-, la accesibilidad (fomentando la instalación de actividades económicas en las nuevas áreas industriales, aprovechando la llegada de la alta velocidad ferroviaria al núcleo...), la centralidad comarcal -mejorada con la dinamización de los servicios sociales y las actividades comerciales- y la calidad de vida urbana, en función de las indicaciones de la Agenda 21 Local, en lo referente a sostenibilidad social, económica y ambiental. Finalmente, también ha de valorarse positivamente la apuesta por la participación local; de hecho, el IX Congreso Nacional de Medio Ambiente (CONAMA) ha premiado al municipio con un accésit en la categoría de municipios entre 20.000 y 50.000 habitantes por el modelo de participación ciudadana asociada al Plan Medina 21.

En definitiva, dentro de las respuestas de las ciudades medias a los desafíos socioeconómicos y territoriales, el ejemplo de Medina del Campo resulta innovador por el papel de liderazgo de su administración local. Los resultados de esta apuesta aún son incipientes sobre el territorio, si bien la generación de una cultura de la participación y la concertación está resultando esencial en la cualificación integral del capital social, elemento básico para la competitividad de ciudades medias.

\section{CONCLUSIONES}

A pesar del carácter excepcional de la alta capacidad innovadora de una pequeña parte del tejido empresarial local en las ciudades medias del nivel apuntado, los casos analizados vienen a confirmar que el conjunto de debilidades que caracteriza a la estructura productiva regional tales como la baja cultura innovadora en el sector empresarial, y una escasa interacción entre empresas y centros de investigación en actividades de $\mathrm{I}+\mathrm{D}$ se hacen más evidentes en lo que podemos denominar los bordes del territorio innovador, es decir, en aquellas medianas o pequeñas ciudades en las que, pese a la importancia de la intervención institucional, persisten rutinas organizativas poco permeables a la innovación, dado el elevado componente de sectores maduros en su estructura 
productiva y la escasez de iniciativas emprendedoras ligadas al desarrollo de actividades innovadoras.

En este contexto es frecuente que sean dominantes procesos rutinarios y conservadores, en parte debidos a la escasa presión del mercado y singularmente de la demanda laboral, ya que o bien han sido municipios que con anterioridad a la crisis de 2008 se encontraban en una situación de facto equivalente al pleno empleo, o bien sus excedentes, especialmente los más cualificados, eran y son aún drenados -al menos así sucede en la última década- hacia los grandes sumideros de actividad y desarrollo que, en la escala provincial-regional son las cuatro mayores capitales de provincia -Valladolid, León Salamanca y Burgosy que a escala supraregional, por su proximidad y dinamismo, es la comunidad de Madrid.

Las alternativas a esta situación, al margen de algunas iniciativas empresariales estrechamente vinculadas a los grandes objetivos de $\mathrm{I}+\mathrm{D}$, impulsadas y parcialmente financiadas por el gobierno regional a través de ADE, se han apoyado o bien - como sucede en Medina del Campo, en una estrecha interrelación entre planificación estratégica y modelos de gobernanza participativa, traducida en la génesis de una densa red social entre agentes, políticas y acciones, o bien como en Aranda de Duero en una destacada presencia de asociaciones empresariales lideradas por Ademar, así como en la participación en redes temáticas de ciudades vinculadas a la explotación del potencial endógeno comarcal.

En ambos casos se ha demostrado el relevante significado de las estrategias de aprendizaje en innovación de las que participan gran parte de los agentes implicados en el funcionamiento del sistema productivo local. Gracias a ellas se ha mantenido la base del tejido empresarial local y se han explorado nuevas áreas de actividad y negocio en los sectores de la energía y alimentación que ya tienen asiento en los nuevos polígonos industriales de Aranda de Duero -Prado Marina- y Medina del Campo - Francisco Lobato, Medina On y el denominado Polígono Escaparate-. De este modo dos ciudades poco vinculadas a las grandes estrategias de $\mathrm{I}+\mathrm{D}$ regionales han podido desarrollar iniciativas empresariales innovadoras y renovar su base productiva.

\section{BIBLIOGRAFÍA}

CARAVACA, I. (Coord.) (2002): Innovación y territorio. Análisis comparado de sistemas productivos locales en Andalucía. Sevilla, Junta de Andalucía.

CaravacA, I., GonzÁlez, G., Silva, R. (2003): «Redes e innovación socioinstitucional en sistemas productivos locales» Boletín de la $A G E, 36,2003$, pp. 103115.

CARAVACA, I. et AL. (2009) «Trayectorias de innovación, competitividad y desarrollo en tres ciudades de Andalucía», en SAlom, J. y Albertos, J.M. (Eds.) (2009). Re- 
des socio institucionales, estrategias de innovación y desarrollo territorial en España. Universidad de Valencia, pp. 53-172.

CASSON, M. (1995): Essays in the Economics of Trust, Londres, Routledge.

GONZÁlez, G. (2006): Innovación, redes y territorio en Andalucía. Sevilla, Junta de Andalucía.

HIRST, P. (1994): Associative Democracy: New Forms of Economic and Social Governance. Amerherst: University of Massachusetts Press.

JUNTA DE CASTILlA Y LEÓN (2007): Estrategia Regional de Investigación Científica, Desarrollo tecnológico e Innovación de Castilla y León 2007-2013. Valladolid.

LAM, A. (2005): «Organizational Innovation», en J. FAGERBERG, D. MOWERY y R. R. NELSON (eds.), The Oxford Handbook of Innovation, Oxford, Oxford University Press.

LuNDVALL, B. (Ed.) (1992): National systems of innovation: towards a theory on innovation and interactive learning. Londres, Pinter Publishers.

MÉNDEZ, R. (2002): «Innovación y desarrollo territorial: algunos debates teóricos recientes». EURE, vol. XXVIII, 84, pp. 63-84.

MÉNDEZ, R. ET AL. (2006): «Ciudades intermedias y desarrollo territorial en Castilla La Mancha» Xeográfica, 6, pp. 69-83.

PORTER, M. (1998): Ventaja competitiva: creación y sostenimiento de un desempeño superior, México, Compañía Editorial Continental.

Salom, J. (2003): «Innovación y actores locales en los nuevos espacios económicos: un estado de la cuestión», Boletín de la Asociación de Geógrafos Españoles, 36, págs. 7-30.

SAlom, J. y Albertos, J.M., (eds.) (2009): Redes socio institucionales, estrategias de innovación y desarrollo territorial en España. Valencia, Universidad de Valencia. 